\title{
@creative
commons
}

ISSN 2590-9770

The Art of Discrete and Applied Mathematics 1 (2018) \#P2.06

https://doi.org/10.26493/2590-9770.1230.f19

(Also available at http://adam-journal.eu)

\section{Mario Gionfriddo and mixed hypergraph coloring}

\author{
Vitaly Voloshin \\ Troy University, Troy, AL, USA
}

Received 20 December 2017, accepted 28 January 2018, published online 14 July 2019

\begin{abstract}
We give a brief description of the explicit and implicit contribution of Mario Gionfriddo to mixed hypergraph coloring.
\end{abstract}

Keywords: Graph and hypergraph coloring, mixed hypergraphs, block designs, Steiner systems.

Math. Subj. Class.: 05C15, 05C65

\section{A little bit of history}

It was the summer of 1992 in new independent state, Republic of Moldova, the very fresh ex-USSR country. Living in Kishinev, the capital city, and desperately looking for any contacts with western mathematicians, one day I went to the library of the Institute of Mathematics and Informatics. That library was good because one could find some additional western mathematical journals compared to the university library. It was an absolutely random event (or was it?): my attention was attracted by the International Mathematical Union Canberra Circular (from Australia!), with the list of mathematical conferences all over the world. At that time the internet was not widely accessible, there was no Google, not even email available. I noticed a very brief, just one paragraph, advertisement that there will be Catania Combinatorial Conference in October 1992, in Italy. And the address of Mario Gionfriddo was simply provided as the contact information.

Since I had nothing to lose, I decided to write a postcard (see both sides in Figure 1). I wrote:

Dear Professor Mario Gionfriddo:

I would be much obliged to you if you could send me invitation/program/information/ Proceedings of the 3rd Catania Combinatorial Conference. I am a specialist in Graph and Hypergraph Theory, Assistant Professor of the Moldova State University, Kishinev.

E-mail address: vvoloshin@troy.edu (Vitaly Voloshin)

(a) (i) This work is licensed under http://creativecommons.org/licenses/by/3.0/ 
I would like to be friend with you. My report may be entitled: "Conditional Colourings on Hypergraphs".

Thank you very much.

Sincerely yours V. Voloshin

24 July 1992.

Since I was studying English from zero at that time (my foreign language was French), writing in English was a good exercise. If you look at this postcard, you may also recognize an old Soviet postcard with the stamps of Republic of Moldova over it. But it was beyond any imagination, what significant events were implied by this simple postcard.

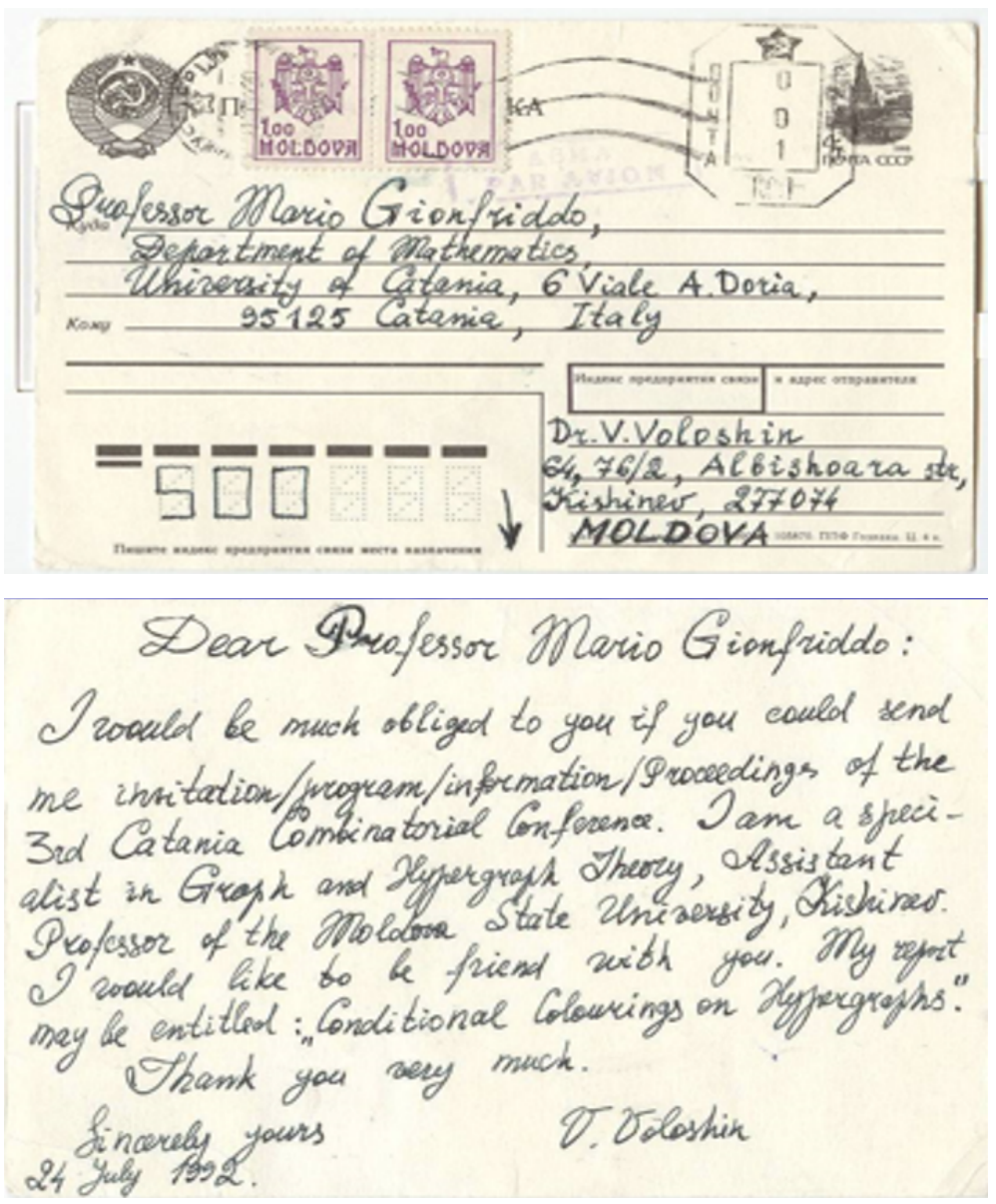

Figure 1: Postcard that changed the world.

I was waiting the reply for about two months and, having none, thought that it was one of many cases left without any answer. All of the sudden, one week before the conference, the invitation letter by Mario Gionfriddo arrived. There was no possibility for me to arrange everything (visa etc.) on such short notice (later I learned it required 1-4 months!). So I had to answer that I can't come. Then Mario asked me for my CV (correspondence by 
email just started in Moldova); it was mailed to Catania. Since then I had no news for a long time and decided that I have to forget it again.

But I will never forget the day of June 26, 1993 when I have received the official invitation by Mario Gionfriddo saying that CNR of Italy has awarded a research grant to me for visiting University of Catania for two months. It was a huge event because it gave me some hope that I can probably survive the hardships of that period. I must confess that there was time when I believed that as mathematician I will not survive. 1992 was the year of war in Moldova, and it felt like we just escaped the Titanic. After arranging multiple problems (like visa, ticket etc.) I arrived to Catania in early October, 1993. I could only recognize Mario at Catania railway station (we never met!) because he was holding the famous book of Claude Berge "Hypergraphs" [3]. It was the best "password" in that moment; and it was the very first application of hypergraph theory in real life for me.

When we started discussions about possible research collaboration, I realized that we have very different backgrounds and not that much in common. Mario mostly worked in block designs but I was not familiar with them except occasional mentioning in the book of Berge. However, at that time I already was developing basic concepts of mixed hypergraph coloring. The basic idea of it was to allow edges that can be monochromatic but must not be polychromatic (all vertices $=$ different colors). At the very beginning they were called "anti-edges" because this term exactly reflected the meaning. Mixing classic edges (non monochromatic subsets) with anti-edges (non polychromatic subsets) lead to the concept of mixed hypergraph coloring. It was completely new at that time (even this circumstance became clear much later!). The very first paper [33] was just published but nobody heard about it in Italy. The main paper [34] was in progress and not even published yet. But because of the generality of graph coloring, the ideas for collaboration came naturally.

It was due to Mario himself and his colleagues Salvatore Milici, Gaetano Quattrocchi, Angelo Lizzio and others. It was due to the Mario's seminar, multiple discussions with international visitors like Zsolt Tuza, Alex Rosa, Curt Lindner, Chris Rodger, Carsten Thomassen, Robin Wilson, and graduate students like Lorenzo Milazzo and many others. Very soon I realized that I got into the very best environment that a mathematician can dream: the international center of active research in graphs, hypergraphs and designs under the leadership of Mario Gionfriddo. The new direction of research aiming at application of mixed hypergraph coloring in coloring of block designs has taken off. It was a matter of not one, not even two years of research collaboration when the very first significant results have been obtained and published.

As I recall, the very first fundamental questions which were worth to work on, were these:

1. What is the upper chromatic number of Steiner triple system (abbreviated by STS for short) considered as $C$-hypergraph? That is, what is the maximum total number of colors when coloring the vertices of each block with at most two colors?

At that time, the STS were colored in old classic way: no block was monochromatic. Under this constraint, the problem on maximum number of colors did not exist since the coloring with $n$ (number of points, or vertices in STS) colors was always feasible.

2. If we color each block with precisely two colors, are there uncolorable Steiner triple systems? That is, are there STS which cannot be colored in this way with any number of colors? 
Notice that in classic coloring, all systems were trivially colorable with $n$ colors and therefore this problem never arose. However, if the system is colorable under these new constraints, then such concepts as the minimum and maximum number of colors naturally arise; they are called the lower and upper chromatic numbers and denoted by $\chi$ and $\bar{\chi}$ respectively.

3 . Is the chromatic spectrum of any STS continuous? That is, whether there exist colorings using any intermediate number of colors between $\chi$ and $\bar{\chi}$. Otherwise there is a gap in chromatic spectrum meaning there is no coloring with a number of colors $k$ such that $\chi<k<\bar{\chi}$.

When I arrived to Catania for the first time, there were a few preliminary results in the first and second questions regarding some other hypergraph classes like interval mixed hypergraphs. But there was no idea, no approach, not even one fact of any mixed hypergraph with the gap in chromatic spectrum.

Simultaneously, in 1993, in order to find out if the concept of mixed hypergraph coloring was new, I submitted the current version of [34] to Paul Erdős with only one this question. The assumption was that Erdős knew everything; and, to my great satisfaction, the answer was yes. In 1993-1996, I received six letters from Paul Erdős, and in the fifth letter he wrote, see Figure 2:

1994 IX 13

Dear Professor Voloshin,

Many thanks for your letter, I hope you will have a pleasant and fruitful time in Catania, please give my regards to Professor Gionfriddo. Keep me informed of your further plans.

Kind regards

Paul Erdôs

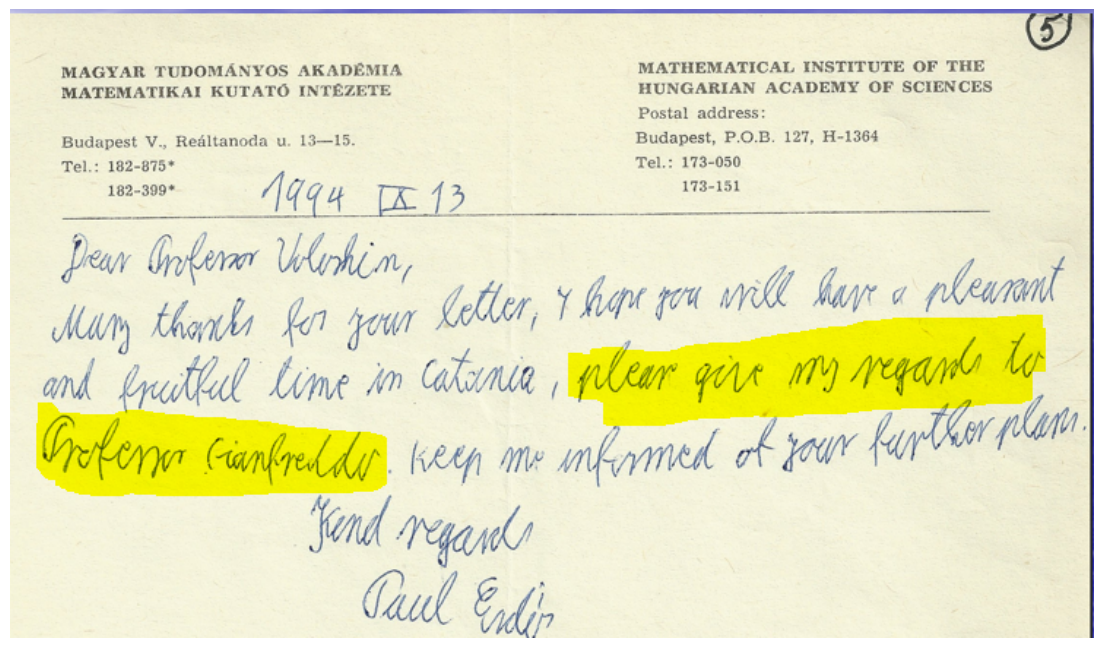

Figure 2: Paul Erdős sends regards to Mario Gionfriddo, 1994.

This letter was an additional evidence of how high was the international recognition of Mario Gionfriddo long before I came to Catania. 


\section{Mathematical results obtained in Catania}

We use the terminology from [4]. Let $V=\left\{v_{1}, v_{2}, \ldots, v_{n}\right\}$ be a finite set of elements called vertices, and let $\mathcal{E}=\left\{E_{1}, E_{2}, \ldots, E_{m}\right\}$ be a family of subsets of $V$ called edges or hyperedges. The pair $\mathcal{H}=(V, \mathcal{E})$ is called a hypergraph with vertex set $V=V(\mathcal{H})$ and edge-set $\mathcal{E}=\mathcal{E}(\mathcal{H})$. The hypergraph $\mathcal{H}=(V, \mathcal{E})$ is sometimes called a set system. If each edge of a hypergraph contains precisely two vertices, then it is a graph.

If every edge of $\mathcal{H}$ is of size $r$, then $\mathcal{H}$ is called an $r$-uniform hypergraph; evidently, a simple graph is a 2-uniform hypergraph.

Let $\{1,2, \ldots, \lambda\}$ be a set of colors. A proper $\lambda$-coloring of a hypergraph $\mathcal{H}=(V, \mathcal{E})$ is a mapping $c: V \rightarrow\{1,2, \ldots, \lambda\}$ for which every edge $E \in \mathcal{E}$ has at least two vertices of different colors. The number of proper $\lambda$-colorings of $\mathcal{H}$ is a polynomial in $\lambda$; it is denoted by $P(\mathcal{H}, \lambda)$ and is called the chromatic polynomial. The minimum value of $\lambda$ for which there exists a proper $\lambda$-coloring of a hypergraph $\mathcal{H}$ is called the chromatic number of $\mathcal{H}$, denoted by $\chi(\mathcal{H})$. A hypergraph $\mathcal{H}$ is $k$-colorable if $\chi(\mathcal{H}) \leq k$.

The concept of a mixed hypergraph coloring was introduced in [33]. Instead of $\mathcal{H}=$ $(V, \mathcal{E})$, the basic idea was to consider a structure $\mathcal{H}=(V, \mathcal{C}, \mathcal{D})$, termed a mixed hypergraph, with two families of subsets called $\mathcal{C}$-edges and $\mathcal{D}$-edges. By definition, a proper $\lambda$-coloring of a mixed hypergraph $\mathcal{H}=(V, \mathcal{C}, \mathcal{D})$ is a mapping $c: V \rightarrow\{1,2, \ldots, \lambda\}$ for which two conditions hold:

- every $C \in \mathcal{C}$ has at least two vertices of a $\mathcal{C}$ ommon color;

- every $D \in \mathcal{D}$ has at least two vertices of $\mathcal{D}$ ifferent colors.

A mixed hypergraph $\mathcal{H}$ is called colorable if it admits at least one proper coloring; and it is uncolorable if no such colorings exist.

The chromatic spectrum is the vector $\left(r_{1}, r_{2}, \ldots, r_{n}\right)$, where each $r_{k}$ is the number of partitions of the vertex set induced by proper colorings using precisely $k$ colors. A gap in the chromatic spectrum is an integer $k$ for which $\chi(\mathcal{H})<k<\bar{\chi}(\mathcal{H})$ and $r_{k}=0$. A mixed hypergraph $\mathcal{H}=(V, \mathcal{C}, \mathcal{D})$ is called a bihypergraph if the families of $\mathcal{C}$-edges and $\mathcal{D}$-edges coincide, i.e., $\mathcal{C}=\mathcal{D}$.

A Steiner system $\mathrm{S}(t, k, v)$ is a $k$-uniform hypergraph of order $v$, for which each $t$ tuple of vertices is contained in precisely one edge. To mention some examples, a system $\mathrm{S}(2,3, v)$ is a Steiner triple system $\operatorname{STS}(v)$, an $\mathbf{S}(3,4, v)$ is a Steiner quadruple system $\operatorname{SQS}(v)$, and an $\mathrm{S}\left(2, q+1, q^{2}+q+1\right)$ is a finite projective plane of order $q$.

The edges are called blocks. We may view each block as a $\mathcal{C}$-edge (when an $\operatorname{STS}(v)$ is denoted by $\operatorname{CSTS}(v)$ ) or as a bi-edge - that is, a $\mathcal{C}$-edge and a $\mathcal{D}$-edge at the same time (when an $\operatorname{STS}(v)$ is denoted by $\operatorname{BSTS}(v)$ ). The notations $\operatorname{CS}(t, k, v), \operatorname{BS}(t, k, v), \operatorname{CSQS}(v)$ and BSQS $(v)$ are derived for the respective systems in a similar way.

The study of the upper chromatic number in Steiner triple systems started in Catania in 1993 and resulted in a series of publications, see [26, 27, 28, 29, 31, 30, 32]. For example, in [30] the authors proved that

$$
\bar{\chi}(\operatorname{BSTS}(v)) \leq \bar{\chi}(\operatorname{CSTS}(v)) \leq k,
$$

for all $v \leq 2^{k}-1$. This upper bound on $\bar{\chi}$ is tight for all $k \geq 2$, and the systems attaining equality were also characterized. In particular, in them the cardinalities of color classes are powers of 2 . The first $\mathrm{PhD}$ Thesis in this field (and in mixed hypergraph coloring in 
general) was defended under supervision of Mario Gionfriddo by Lorenzo Milazzo in 1997, see [28].

A coloring of a Steiner triple system $\operatorname{STS}(n)$ in a way that every block receives precisely two colors is also called a bicoloring [7]. All bicolorable $\operatorname{STS}\left(2^{h}-1\right)$ s have upper chromatic number $\bar{\chi} \leq h$. If $\bar{\chi}=h<10$, then lower and upper chromatic numbers coincide, i.e., $\chi=\bar{\chi}=h$. In 2003, Mario raised a subtle and challenging conjecture [13] that this equality holds whenever $\bar{\chi}=h \geq 2$. Until today it remains open, intriguing and motivating for further research. Some of the most recent results in this direction discuss extensions of bicolorings of $\operatorname{STS}(v)$ to bicolorings of $\operatorname{STS}(2 v+1)$ obtained by using the so called doubling plus one construction, see [5].

The problem of colorability of BSTS was also first formulated in Catania in 1993 though no example of uncolorable BSTS was found. The first such example was constructed by Ganter at TU Dresden, and all uncolorable BSTS(15)s have been characterized by Rosa [35]: BSTS(15) is colorable if and only if it contains BSTS(7) as a subsystem. Out of the 80 non-isomorphic BSTS(15)s, only 23 meet this criterion and are therefore colorable. The other 57 are uncolorable. It follows that uncolorable $\operatorname{BSTS}(n)$ s exist for each admissible $n \geq 15$.

As to BSQS, the situation is much more difficult. Even though the problem to find at least one uncolorable BSQS was formulated first in Catania in 1993 as well, no one such system has been found. The conjecture is that they exist. The best result related to this problem is by Lo Faro, Milazzo and Tripodi [22]: all BSQS $(n)$ are colorable for all admissible $n \leq 16$. Therefore, the smallest admissible $n$ for which uncolorable $\operatorname{BSTS}(n)$ may exist is $n=20$.

There is a significant series of important results and publications by the whole Catania group of mathematicians like these $[2,6,13,14,15,16,17,20,22,23,24,25,26,27,28$, 29, 31, 30, 32] just to name a few. Here is the right point to mention that Catania group is very closely related to Messina group of mathematicians who work in the same direction: Giovanni Lo Faro, Enzo Li Marzi, Corinna Marino and Antoinette Tripodi. Because of this connection, as one can see, they also have published many papers generally speaking in mixed hypergraph coloring.

It was October of 1998. After half year stay in USA, I arrived to Catania where I met Zsolt Tuza as usual, again. We were working on some problems when I received an email from Dhruv Mubayi, a graduate student of Doug West, University of Illinois at Urbana-Champaign. In that email, Dhruv communicated that while looking at a completely different problem, he found an example of mixed hypergraph on 16 vertices, $36 \mathcal{D}$-edges and 144 (!) $\mathcal{C}$-edges with the gap in the chromatic spectrum. It was a shocking discovery, literally a breakthrough! As I recall, when we realized it, we immediately started searching for the smallest example, and it took just one night for Zsolt to come up next morning with the example depicted in Figure 3. It had 6 vertices $\{1,2,3,4,5,6\}, 2 \mathcal{D}$-edges $\{1,6\}$ and $\{2,3,4,5\}$ ) (solid line and curve), $3 \mathcal{C}$-edges $\{2,3,4\},\{3,4,5\}$ and $\{2,4,5\}$ (dashed curves), and 4 bi-edges $\{1,2,3\},\{1,4,5\},\{6,2,3\}$, and $\{6,4,5\}$ (bold curves). One can easily see that if vertices 2 and 3 are colored with the same color, say $A$, then this coloring extends in a unique way to vertices 4 and 5 with color $B$, vertex 1 with color $C$ and vertex 6 with color $D$. If, on the contrary, vertices 2 and 3 are colored differently, say colors $A$ and $B$, then this coloring extends to vertex 1 with color $A$, vertex 4 with color $A$, and vertices 5 , 6 with color $B$. Actually, there are four distinct extensions of this coloring, all with colors $A$ and $B$. So, there is no coloring using 3 colors, and the chromatic spectrum of this example 
is $R(\mathcal{H})=(0,4,0,1,0,0)$. The chromatic polynomial $P(\mathcal{H}, \lambda)=\lambda(\lambda-1)\left(\lambda^{2}-5 \lambda+10\right)$.

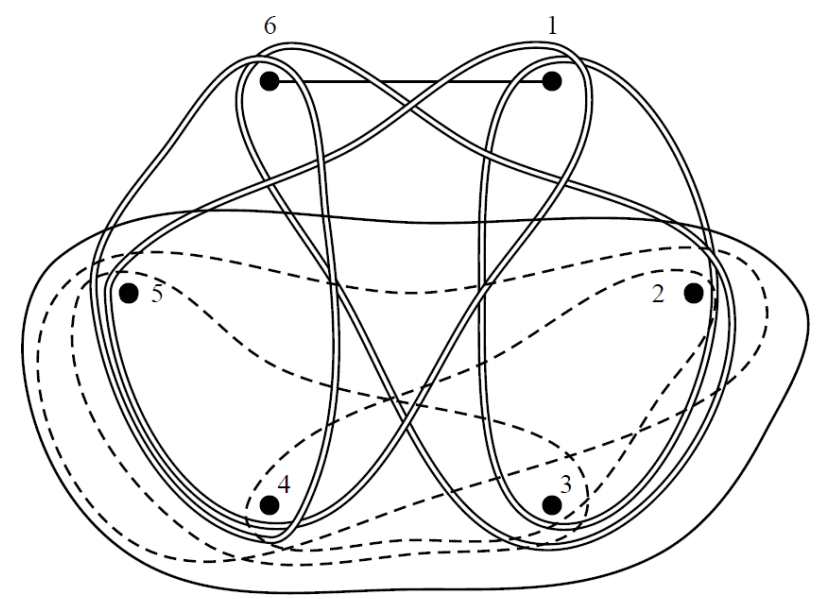

Figure 3: The very first smallest example with gap, Catania, 1998.

The first results have been published in [21]. It was the very beginning of the chase for the gaps in the chromatic spectrum in mixed hypergraphs which with variable success continues until today.

Around the year of 2000, Catania group was reinforced by new researcher, Lucia Gionfriddo. After defending PhD Thesis, she got interested in problems related to mixed hypergraph coloring, namely the gaps in the chromatic spectrum. For an impressively short period of time, Lucia has discovered the first designs, namely $P_{3}$-designs with the gaps in the chromatic spectrum. The idea was to consider decompositions of complete graphs into $P_{3}$ (a path on 3 vertices) and declare every block as a bi-edge, i.e., colorable with precisely two colors. It is a special case of bi-hypergraph. She proved that there are many such structures with many gaps, in particular, big gaps, even gaps, odd gaps, etc., see [8, 9, 10, 11$]$. Later other designs, namely $P_{4}$-designs with the gaps have been found when considering equicolorings in [1]. Surprisingly, until today, these are the only examples of block designs with the gaps. We do not know anything about continuity of the chromatic spectrum of BSTS or BSQS, for example.

Based on Lucia's results, while my stay in Catania, we were able to carry out some computational experiments and construct the smallest 3-uniform bi-hypergraph with the gap in the chromatic spectrum, see Figure 4: it contains 7 vertices and 9 bi-edges and its chromatic spectrum is $R(\mathcal{H})=(0,12,0,3,0,0,0)$, see [12]. The chromatic polynomial of this bi-hypergraph $P(\mathcal{H}, \lambda)=3 \lambda(\lambda-1)\left(\lambda^{2}-5 \lambda+10\right)$. It is interesting that the chromatic spectra and chromatic polynomials of hypergraphs in Figure 3 and in Figure 4 are related (compare). They were found independently.

There is one more result that is worth to mention. It is about the upper chromatic index of a multi-graph, which represents a type of anti-Vizing theorem. It was first formulated in [34] as Problem 13 and was implied by the duality of mixed hypergraphs. Consider the colorings of the edges of a multi-graph in such a way that every non-pendant vertex is incident to at least two edges of the same color. The maximum number of colors that can be used in such colorings is the upper chromatic index of a multi-graph $G$, denoted 


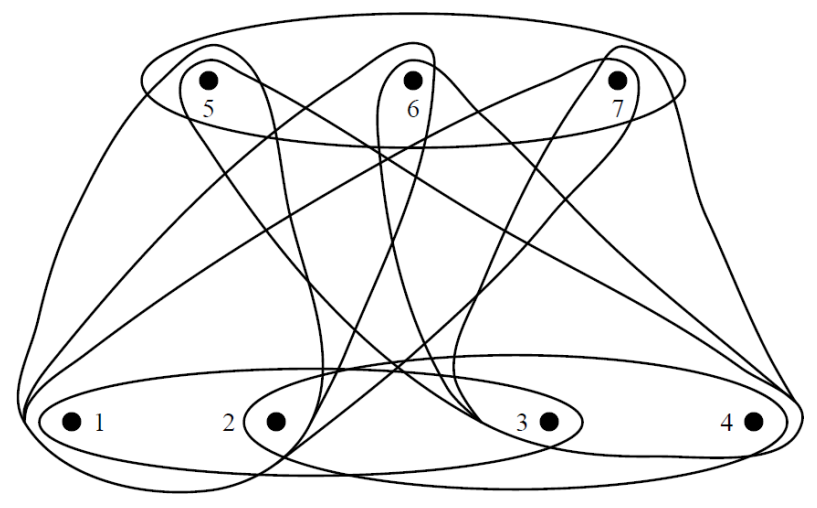

Figure 4: The smallest 3-uniform example with gap, Catania, 2002.

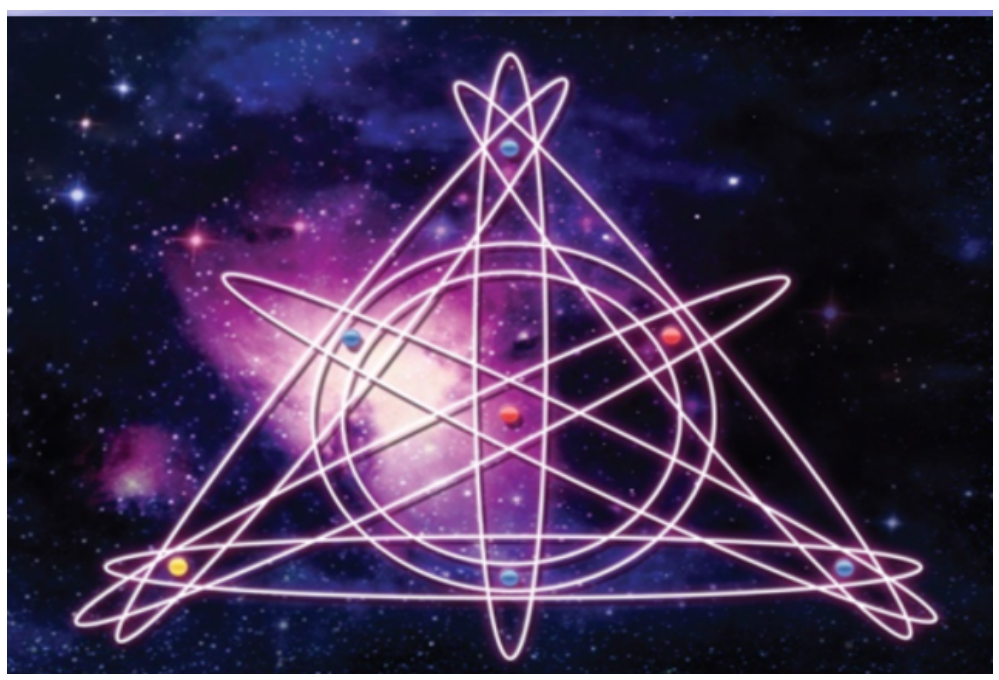

Figure 5: Mixed Hypergraph Coloring encoded in BSTS(7). 
by $\bar{\chi}^{\prime}(G)$. The exact value of it was found in [18]. It was proved that if a multi-graph $G$ has $n$ vertices, $m$ edges, $p$ pendant vertices and maximum number $c$ of disjoint cycles, then $\bar{\chi}^{\prime}(G)=c+m-n+p$. This result was reported by Lorenzo Milazzo at the Second Lethbridge Workshop on Designs, Codes, Cryptography and Graph Theory, July $9-14$, 2001.

One of the basic results in applications of mixed hypergraph coloring to block designs, namely, about the cardinality of color classes being powers of 2 in the optimal coloring of $\operatorname{BSTS}(7)$, was encoded in the picture of it on the book cover of [19]: one vertex in yellow color, two vertices in red color and four vertices in blue, see Figure 5. In contrast to classic drawing, it is depicted as a hypergraph. Every block is colored with two colors and any other proper coloring has the same distribution of color classes.

\section{Conclusion}

In conclusion, what I witnessed through decades, was a multilateral activity by Mario Gionfriddo which can be summarized in this way (I do not pretend to be complete):

Mario Gionfriddo:

1. Created an outstanding scientific school of researchers in Graphs, Hypergraphs and Designs with many publications in top journals all over the world.

2. Turned University of Catania and University of Messina into major centers of international collaboration. Proved that he is a great teacher, educator, researcher, organizer, and in general, a great leader in contemporary mathematics.

3. Played and still plays an outstanding role in Italian and especially Sicilian Discrete Mathematics, namely, the Theory of Graphs, Hypergraphs and Designs.

4. Played an outstanding explicit and implicit role in developing the theory of Mixed Hypergraph Coloring. Explicit: by personal participation in research and actually proving many theorems. Implicit: by inviting researchers and organizing seminars, workshops and conferences where actual collaboration occurred.

\section{Dear Mario,}

I congratulate you on the occasion of 70th anniversary, thank you for your great role in my life and wish you a good health and further achievements in developing Graphs, Hypergraphs and Designs!

Remark. Dear reader! If it were not that postcard, randomly mailed in 1992 (Figure 1), at this very same moment you would read a very different paper. 


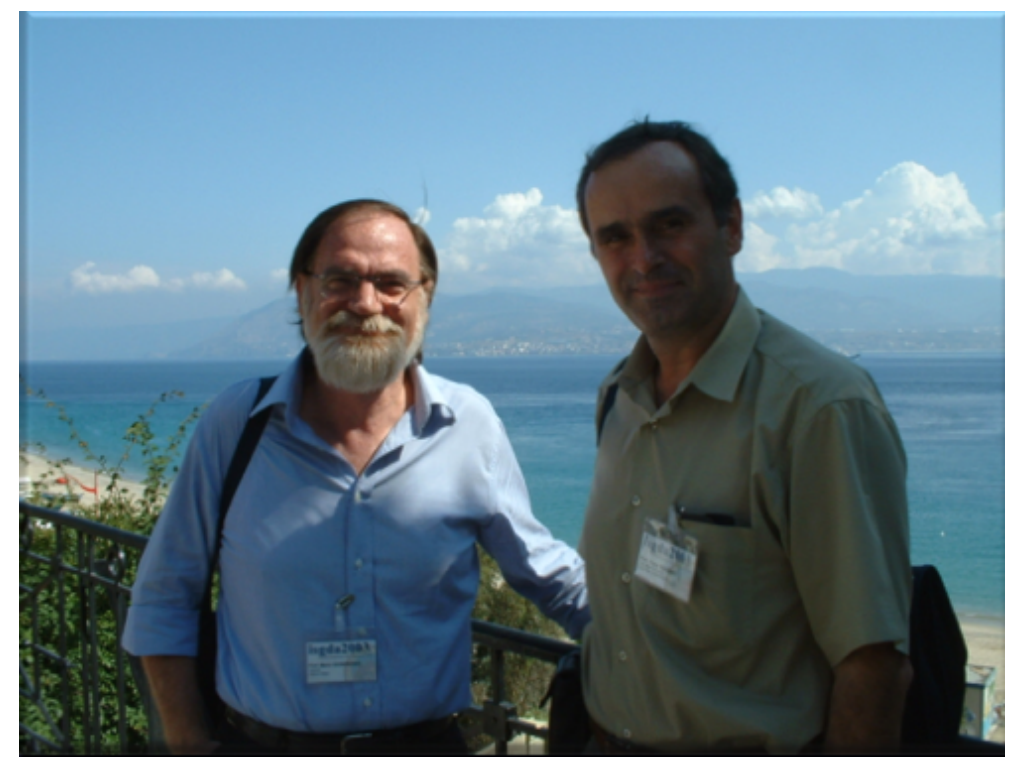

Figure 6: With Mario Gionfriddo, Messina, 2003.

\section{References}

[1] A. Amato, M. Gionfriddo and L. Milazzo, 2-regular equicolourings for $\mathbf{P}_{4}$-designs, Discrete Math. 312 (2012), 2252-2261, doi:10.1016/j.disc.2012.03.030.

[2] A. Amato, M. Gionfriddo and L. Milazzo, A survey of Lucia Gionfriddo's results about colourings in $\mathrm{BP}_{3}$-designs, in: M. Buratti, C. Lindner, F. Mazzocca and N. Melone (eds.), Recent Results in Designs and Graphs: A Tribute to Lucia Gionfriddo, Aracne, Rome, volume 28 of Quaderni di Matematica, pp. 39-60, 2012.

[3] C. Berge, Hypergraphs: Combinatorics of Finite Sets, volume 45 of North-Holland Mathematical Library, North-Holland, Amsterdam, 1989.

[4] Cs. Bujtás, Zs. Tuza and V. Voloshin, Hypergraph colouring, in: L. W. Beineke and R. J. Wilson (eds.), Topics in Chromatic Graph Theory, Cambridge Univiversity Press, Cambridge, volume 156 of Encyclopedia of Mathematics and its Applications, pp. 230-254, 2015, doi: 10.1017/cbo9781139519793.014.

[5] Cs. Bujtás, M. Gionfriddo, E. Guardo, L. Milazzo, Zs. Tuza and V. Voloshin, Extended bicolorings of Steiner triple systems of order $2^{h}-1$, Taiwanese J. Math. 21 (2017), 1265-1276, doi:10.11650/tjm/8042.

[6] M. Buratti, M. Gionfriddo, L. Milazzo and V. Voloshin, Lower and upper chromatic numbers for BSTSs $\left(2^{h}-1\right)$, Comput. Sci. J. Moldova 9 (2001), 259-272, http : / /www . math.md/ publications/csjm/issues/v9-n2/8294/.

[7] C. J. Colbourn, J. H. Dinitz and A. Rosa, Bicoloring Steiner triple systems, Electron. J. Combin. 6 (1999), \#R25 (16 pages), https: / /www. combinatorics.org/ojs/index.php/ eljc/article/view/v6ilr25.

[8] L. Gionfriddo, Extremal gaps in BP3-designs, Comput. Sci. J. Moldova 9 (2001), 305-320, http://www.math.md/publications/csjm/issues/v9-n3/8302/. 
[9] L. Gionfriddo, $P_{3}$-designs with gaps in the chromatic spectrum, Rend. Sem. Mat. Messina Ser. II 8(24) (2001/02), suppl., 49-58.

[10] L. Gionfriddo, Construction of $\mathrm{BP}_{3}$-designs with mononumerical spectrum, Util. Math. 65 (2004), 201-218.

[11] L. Gionfriddo, Voloshin's colourings of $P_{3}$-designs, Discrete Math. 275 (2004), 137-149, doi: 10.1016/s0012-365x(03)00104-3.

[12] L. Gionfriddo and V. Voloshin, The smallest bihypergraph with a gap in the chromatic spectrum has 7 vertices and 9 edges, Bull. Inst. Combin. Appl. 36 (2002), 73-79.

[13] M. Gionfriddo, Colourings of hypergraphs and mixed hypergraphs, Rend. Sem. Mat. Messina Ser. II 9(25) (2003), 87-97.

[14] M. Gionfriddo, E. Guardo and L. Milazzo, Extending bicolorings for Steiner triple systems, Appl. Anal. Discrete Math. 7 (2013), 225-234, doi:10.2298/aadm130827019g.

[15] M. Gionfriddo, E. Guardo, L. Milazzo and V. I. Voloshin, Feasible sets of small bicolorable STSs, Australas. J. Combin. 59 (2014), 107-119, https: / / j jc.maths.uq. edu.au/ pdf/59/ajc_v59_p107.pdf.

[16] M. Gionfriddo, A. Lizzio, L. Milazzo and V. Voloshin, Upper chromatic number and strict colorings of quadruple systems, in: M. Buratti, C. Lindner, F. Mazzocca and N. Melone (eds.), Recent Results in Designs and Graphs: A Tribute to Lucia Gionfriddo, Aracne, Rome, volume 28 of Quaderni di Matematica, pp. 339-349, 2012.

[17] M. Gionfriddo, L. Milazzo, A. Rosa and V. Voloshin, Bicolouring Steiner systems $S(2,4, v)$, Discrete Math. 283 (2004), 249-253, doi:10.1016/j.disc.2003.11.016.

[18] M. Gionfriddo, L. Milazzo and V. Voloshin, On the upper chromatic index of a multigraph, Comput. Sci. J. Moldova 10 (2002), 81-91, http: / /www. math.md/publications/ Cs jm/issues/v10-n1/8333/.

[19] M. Gionfriddo, L. Milazzo and V. Voloshin, Hypergraphs and Designs, Mathematics Research Developments, Nova Science Publishers, New York, 2014.

[20] T. Griggs, G. Lo Faro and G. Quattrocchi, On some colouring of 4-cycle systems with specified block colour patterns, Discrete Math. 308 (2008), 465-478, doi:10.1016/j.disc.2006.11.063.

[21] T. Jiang, D. Mubayi, Zs. Tuza, V. Voloshin and D. B. West, The chromatic spectrum of mixed hypergraphs, Graphs Combin. 18 (2002), 309-318, doi:10.1007/s003730200023.

[22] G. Lo Faro, L. Milazzo and A. Tripodi, The first BSTS with different upper and lower chromatic numbers, Australas. J. Combin. 22 (2000), 123-133, https: / / a jc . maths . uq. edu . au/ pdf/22/ocr-ajc-v22-p123.pdf.

[23] G. Lo Faro, L. Milazzo and A. Tripodi, On the upper and lower chromatic numbers of BSQSs(16), Electron. J. Combin. 8 (2001), \#R6, (8 pages), https://www . combinatorics.org/ojs/index.php/eljc/article/view/v8ilr6.

[24] G. Lo Faro and A. Tripodi, Triplication for BSTSs and uncolourability, Discrete Math. 284 (2004), 197-203, doi:10.1016/j.disc.2003.11.032.

[25] G. Lo Faro and A. Tripodi, Strict colourings of STS $(3 v) \mathrm{s}$ and uncolourable BSTS $(3 v) \mathrm{s}$, Discrete Math. 301 (2005), 117-123, doi:10.1016/j.disc.2004.11.024.

[26] L. Milazzo, On upper chromatic number for SQS(10) and SQS(16), Matematiche (Catania) 50 (1995), 179-193, https: / / lematematiche.dmi.unict.it/index.php/ lematematiche/article/view/508.

[27] L. Milazzo, The monochromatic block number, Discrete Math. 165/166 (1997), 487-496, doi: 10.1016/s0012-365x(96)00195-1. 
[28] L. Milazzo, Sul numero cromatico superiore nei sistemi di Steiner, Ph.D. thesis, University of Catania, 1997.

[29] L. Milazzo, Strict colorings for Steiner systems of STS and SQS type, Rend. Sem. Mat. Messina Ser. II 5(21) (1999), suppl., 107-117.

[30] L. Milazzo and Zs. Tuza, Upper chromatic number of Steiner triple and quadruple systems, Discrete Math. 174 (1997), 247-259, doi:10.1016/s0012-365x(97)80332-9.

[31] L. Milazzo and Zs. Tuza, Strict colourings for classes of Steiner triple systems, Discrete Math. 182 (1998), 233-243, doi:10.1016/s0012-365x(97)00143-x.

[32] S. Milici, A. Rosa and V. Voloshin, Colouring Steiner systems with specified block colour patterns, Discrete Math. 240 (2001), 145-160, doi:10.1016/s0012-365x(00)00388-5.

[33] V. Voloshin, The mixed hypergraphs, Comput. Sci. J. Moldova 1 (1993), 45-52, http: // www. math.md/publications/cs jm/issues/v1-n1/7747/.

[34] V. I. Voloshin, On the upper chromatic number of a hypergraph, Australas. J. Combin. 11 (1995), 25-45, https://ajc.maths.uq.edu.au/pdf/11/ocr-ajc-v11-p25. pdf.

[35] V. I. Voloshin, Coloring Mixed Hypergraphs: Theory, Algorithms and Applications, volume 17 of Fields Institute Monographs, American Mathematical Society, Providence, RI, 2002, doi: 10.1090/fim/017. 